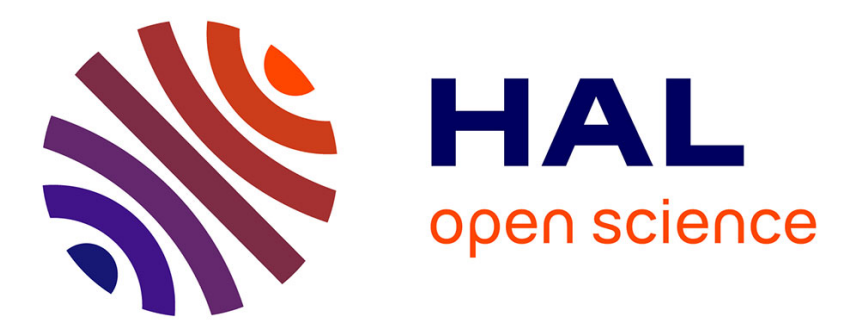

\title{
MCMC-Based Tracking and Identification of Leaders in Groups
}

\author{
Avishy Carmi, Lyudmila Mihaylova, François Septier, Sze Kim Pang, Pini \\ Gurfil, Simon J. Godsill
}

\section{- To cite this version:}

Avishy Carmi, Lyudmila Mihaylova, François Septier, Sze Kim Pang, Pini Gurfil, et al.. MCMCBased Tracking and Identification of Leaders in Groups. IEEE International Conference on Computer Vision Workshops (ICCV Workshops), Nov 2011, Barcelona, Spain. pp.112-119, 10.1109/ICCVW.2011.6130232 . hal-00685921

\section{HAL Id: hal-00685921 https://hal-imt.archives-ouvertes.fr/hal-00685921}

Submitted on 6 Apr 2012

HAL is a multi-disciplinary open access archive for the deposit and dissemination of scientific research documents, whether they are published or not. The documents may come from teaching and research institutions in France or abroad, or from public or private research centers.
L'archive ouverte pluridisciplinaire HAL, est destinée au dépôt et à la diffusion de documents scientifiques de niveau recherche, publiés ou non, émanant des établissements d'enseignement et de recherche français ou étrangers, des laboratoires publics ou privés. 


\title{
MCMC-Based Tracking and Identification of Leaders in Groups
}

\author{
Avishy Y. Carmi， Lyudmila Mihaylova, François Septier, Sze Kim Pang, \\ Pini Gurfil and Simon J. Godsill
}

\begin{abstract}
We present a novel framework for identifying and tracking dominant agents in groups. Our proposed approach relies on a causality detection scheme that is capable of ranking agents with respect to their contribution in shaping the system's collective behaviour based exclusively on the agents' observed trajectories. Further, the reasoning paradigm is made robust to multiple emissions and clutter by employing a class of recently introduced Markov chain Monte Carlo-based group tracking methods. Examples are provided that demonstrate the strong potential of the proposed scheme in identifying actual leaders in swarms of interacting agents and moving crowds.
\end{abstract}

\section{Introduction}

In recent years there has been an increasing interest in tracking a number of interacting objects moving in a coordinated fashion. Such situations are frequently encountered in many fields, such as video surveillance, feature tracking in video sequences, biomedicine, neuroscience and meteorology, to mention only a few. Although individual objects in the group can exhibit independent movement at a certain level, overall the group will move as one whole, by synchronizing the movement of the individual entities and avoiding collisions. In most of the multi-object tracking methods, as opposed to groups tracking methods, tracking of individual objects is the common approach.

It is clear that inference in such environments have to cope with an ever growing complexity induced by the spatio-temporal interrelations among individuals in the

Avishy Y. Carmi is with the Department of Mechanical and Aerospace Engineering, Nanyang Technological University, Singapore , acarmi@ntu.edu.sg, Lyudmila Mihaylova is with the School of Computing and Communications, Lancaster University, United Kingdom, mila.mihaylova@lancaster.ac.uk, François Septier is with the Signal Processing and Information Theory Group, TELECOM Lille 1, France, francois.septier@telecom-lille1.eu, Sze Kim Pang is with the DSO National Laboratories, Singapore, pszekim@dso.org.sg, Pini Gurfil is with the Department of Aerospace Engineering, Technion - Israel Institute of Technology, Israel, pgurfil@technion.ac.il, Simon J. Godsill is with the Department of Engineering, University of Cambridge, United Kingdom, sjg@eng.cam.ac.uk scene. In some cases where the number of objects becomes excessively large, it might be impractical to track them all individually (e.g., based on image features). An efficient approach would then consist of tracking the clustering structure formed by object concentrations rather than individual entities.

Groups are often referred to as structured objects, a term which reflects the ingrained interplay between their components. These endogenous forces give rise to group hierarchies and are instrumental in producing emergent phenomena. Fortunately, these are exactly the factors essential for maintaining coordination within and between groups, a premise which to some extent allows us to treat them as united entities in a high level tracking paradigm. Any knowledge of existence of such interrelations facilitates sophisticated agent-based behavioural modeling which, in practice, comprises of a set of local interaction rules or mutually interacting processes (e.g., Boids system [21], causality models $[12,20])$ - an approach which by itself provides insightful justifications of characteristic behaviours in the fundamental subsystem level and likewise of group hierarchies and emergent social patterns (see [20]).

\subsection{Reasoning About Behavioural Traits}

Being the underlying driving mechanism for evoking emergent phenomena, hierarchies and principal behaviour patterns, the ingrained interactions between agents are possibly the most pivotal factors that should be scrutinized in high level scene understanding. Such interrelations can take the form of a causal chain in which an agent's decisions and behaviour are affected by its neighbours and likewise have either direct or indirect influence on other agents. The ability to fully represent these interrelations based exclusively on passive observations such as velocity and position, lays the ground for the development of sophisticated reasoning schemes that can potentially be used in applications such as activity detection, intentionality prediction, and artificial awareness.

In this work we demonstrate this concept by developing a causality reasoning framework for ranking agents with respect to their cumulative contribution in shaping the collective behaviour of the system. In particular, our framework is 
able to distinguish leaders and followers based exclusively on their observed trajectories.

\subsection{Novelties and Contributions}

The contribution of this work is twofold. Firstly, a novel causality reasoning scheme is derived for ranking agents with respect to their decision-making capabilities (dominance) as substantiated by the observed emergent behaviour. Dominant agents in that sense are considered to have a prominent influence on the collective behaviour and are experimentally shown to coincide with actual leaders in groups. Secondly, the causality scheme is consolidated with a recently introduced Markov chain Monte Carlo (MCMC)based particle method $[6,19]$ for tracking agents and group hierarchies in potentially cluttered environments.

The subsequent sections $(1.3-2)$ provide an overview of existing group tracking schemes with an emphasis on the underlying MCMC-based particle methods.

The remaining part of this paper is organised as follows. Section 3 develops the causality-driven agent ranking approach. Section 4 demonstrates the performance of the causality identification scheme using a few illustrative examples. Finally, concluding remarks and some open issues are discussed in Section 5.

\subsection{Multiple Group Tracking}

Over the past decade various methods have been developed for group tracking. These can be divided into two broad classes, depending on the underlying complexities: 1) methods for a relatively small number of groups, with a small number of group components [10,16, 19], and 2) methods for groups comprised of hundreds or thousands of objects (normally referred to as cluster/crowd tracking techniques) $[2,6]$. In the second case the whole group is usually considered as an extended object (an ellipse or a circle) which centre position is estimated, together with the parameters of the extent.

Different models of groups of objects have been proposed in the literature, such as particle models for flocks of birds [14], and leader-follower models [17]. However, estimating the dynamic evolution of the group structure has not been widely studied in the literature, although there are similarities with methods used in evolving network models $[1,8]$.

Typically tracking many objects (hundreds or thousands) can be solved by clustering techniques or other methods where the aggregated motion is estimated, as it is in the case of vehicular traffic flow prediction/ estimation, with fluid dynamics type of models combined with particle filtering techniques [18]. For thousands of objects forming a group, the only possible solution is to consider them as an extended object. The extended object tracking problem reduces then to joint state and parameter estimation.
Estimation of parameters in general nonlinear nonGaussian state-space models is a long-standing problem. Since particle filters (PFs) are known with the challenges they face for parameter estimation and for joint state and parameter estimation [4], most solutions in the literature split the problems into two parts: $i$ ) state estimation, followed by ii) parameter estimation (see e.g., [3]). In [3] an extended object tracking problem is solved when the static parameters are estimated using Monte Carlo methods (data augmentation and particle filtering), whereas the states are estimated with a Mixture Kalman filter or with an interacting multiple model filter.

\section{PFs for Tracking in Variable State Dimensions}

An extension of the PF technique to a varying number of objects is introduced in [23], [19] and [16]. In [23] a PF implementation of the PHD filter is derived. This algorithm maintains a representation of the filtering belief mass function using random set realizations (i.e., particles of varying dimensions). The samples are propagated and updated based on a Bayesian recursion consisting of set integrals. Both works of [19] and [16] develop a MCMC PF scheme for tracking varying numbers of interacting objects. The MCMC approach outperforms the conventional PF due to its efficient sampling mechanism. Nevertheless, in its traditional non-sequential form it is inadequate for sequential estimation. The techniques used by [19] and [16] amend the MCMC for sequential filtering (see also [5]). The work in [16] copes with inconsistencies in state dimension by utilizing the reversible jump MCMC method introduced in [13]. In [19], on the other hand the computation of the marginal filtering distribution is avoided as in [5]. The algorithm operates on a fixed dimension state space through indicator variables for labeling of active object states (the two approaches are essentially equivalent).

\section{Models and Algorithms for Group Tracking}

This section briefly reviews the fundamental concepts underlying the MCMC-based group tracking approaches in [19] and [6].

\subsection{Virtual Leader Model}

The idea of group modeling is to adopt a behavioural model in which each member of a group interacts with the other members of the group, typically making its velocity and position more similar to that of others in the same group. In [19], this idea has been conveniently formulated in continuous time through a multivariate stochastic differential equation (SDE) and then derived in discrete time without approximation errors, owing to the assumed linear and Gaussian form of the model. In particular, two different models have been proposed. In the first, the basic group 
model, the group parameter is modeled as a deterministic function of the objects. In the second, the group model with a virtual leader, an additional state variable is introduced in order to model the bulk or group parameter. This second approach is closer in spirit to the bulk velocity model and virtual leader-follower model [17]. Such model provides a more flexible behaviour since the virtual leader is no longer a deterministic function of the individual object states. Figure 1 gives a graphical illustration of the restoring forces towards the virtual leader for a group of five objects.

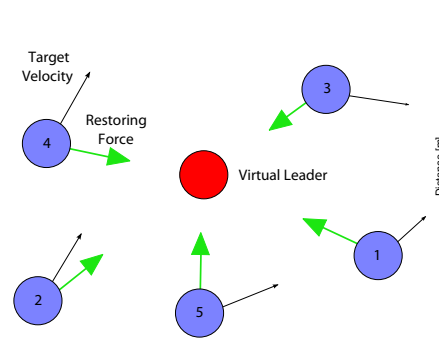

(a)

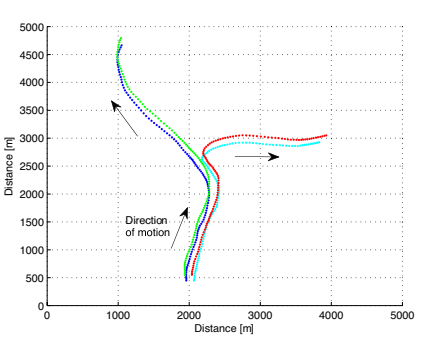

(b)
Figure 1. Group model with virtual leader - Illustration of the restoring forces (a) and of a single realisation showing a group of 4 objects that splits into 2 groups of 2 objects (b).

The spatio-temporal structure for the $i$ th object in a group, as defined in [19], is given by :

$$
\begin{aligned}
d \dot{\boldsymbol{\mu}}_{t, i}^{x}= & \left\{-\alpha\left[\boldsymbol{\mu}_{t, i}^{x}-\boldsymbol{v}_{t}^{x}\right]-\gamma_{1} \dot{\boldsymbol{\mu}}_{t, i}^{x}\right. \\
& \left.-\beta\left[\dot{\boldsymbol{\mu}}_{t, i}^{x}-\dot{\boldsymbol{v}}_{t}^{x}\right]+\boldsymbol{r}_{i}\right\} d t+\sigma_{x} d \boldsymbol{W}_{t, i}^{x} \\
d \dot{\boldsymbol{v}}_{t}^{x}= & -\gamma_{2} \dot{\boldsymbol{v}}_{t}^{x} d t+\sigma_{g} d \boldsymbol{G}_{t}^{x}
\end{aligned}
$$

Here $\boldsymbol{\mu}_{t, i}^{x}$ is the Cartesian position in the $X$ direction of the $i^{t h}$ object in the group at time $t$, with $\dot{\boldsymbol{\mu}}_{t, i}^{x}$ the corresponding velocity. $\boldsymbol{v}_{t}^{x}$ and $\dot{\boldsymbol{v}}_{t}^{x}$ represent respectively the Cartesian position and the velocity both in the $X$ direction of the unobserved virtual leader of the group. $\boldsymbol{W}_{t, i}^{x}$ and $\boldsymbol{G}_{t}^{x}$ are two independent standard Brownian motions. $\boldsymbol{W}_{t, i}^{x}$ is assumed to be independently generated for each object $i$ in the group, whereas $G_{t}^{x}$ is a noise component common to all members of a group. The parameters $\alpha$ and $\beta$ are positive, and reflect the strength of the pull towards the group bulk. The "mean reversion" terms $\gamma_{1} \dot{\boldsymbol{\mu}}_{t, i}^{x}$ and $\gamma_{2} \dot{\boldsymbol{v}}_{t}^{x}$ simply prevent the velocities of the object and the virtual leader drifting up to very large values with time. Finally, in order to reduce or eliminate behaviour in which objects become colocated or collide spatially, which are clearly infeasible or highly unlikely in practice, an additional repulsive force $\boldsymbol{r}_{i}$ is introduced in (1) when objects become too close.

\subsubsection{Modeling Groups of Extended Objects}

In practice, objects may produce more than a single emission, and in some cases they may indeed consist of many individual entities moving in a coordinated fashion (i.e., clusters). Such scenarios normally involve additional extent parameters that embody the potentially dynamic physical boundary of an object. In this respect, the fairly simple idea adopted in [6] represents a dynamically evolving group of extended objects by means of a time-varying Gaussian mixture model (i.e., each mixture component corresponds to an individual object). The underlying properties of the instantaneous clustering configuration, namely, the number of mixture components, their associated weights, means and covariances, are then estimated exclusively from the possibly cluttered and noisy emissions.

\subsection{Sequential Inference Using MCMC-Based PF}

The group tracking problems discussed above can be efficiently solved via the MCMC-based particle method initially proposed for solution of group tracking problems in [19]. This method aims at sequentially approximating the following joint posterior distribution

$$
p\left(\mathbf{x}_{k}, \mathbf{x}_{k-1} \mid \mathbf{z}_{0: k}\right) \propto p\left(\mathbf{z}_{k} \mid \mathbf{x}_{k}\right) p\left(\mathbf{x}_{k} \mid \mathbf{x}_{k-1}\right) p\left(\mathbf{x}_{k-1} \mid \mathbf{z}_{0: k-1}\right)
$$

where the state vector $\mathbf{x}_{\mathbf{k}}$ comprises of the objects' instantaneous position, velocity and extent parameters at time $t_{k}$, and $\mathbf{z}_{0: k}=\left\{\mathbf{z}_{0}, \ldots, \mathbf{z}_{k}\right\}$ is the observation history up to time $t_{k}$. In what follows we would refer to the (discrete) time $t_{k}$ as simply $k$.

Since the closed form expression of the distribution $p\left(\mathbf{x}_{k-1} \mid \mathbf{z}_{0: k-1}\right)$ is generally unknown, the proposed scheme approximates it by using a set of unweighted particles

$$
p\left(\mathbf{x}_{k-1} \mid \mathbf{z}_{0: k-1}\right) \approx \frac{1}{N_{p}} \sum_{j=1}^{N_{p}} \delta\left(\mathbf{x}_{k-1}-\mathbf{x}_{k-1}^{(j)}\right)
$$

where $N_{p}$ is the number of particles and $(j)$ is the particle index. Then, by plugging this particle approximation into (3), an appropriate MCMC scheme can be used to draw from the joint posterior distribution $p\left(\mathbf{x}_{k}, \mathbf{x}_{k-1} \mid \mathbf{z}_{0: k}\right)$. The converged MCMC outputs are then extracted to give an empirical approximation of the posterior distribution of interest at time $k$, thus seeding the next step of the filtering at time $k+1$.

At the $m$ th MCMC iteration, the following procedure is performed to obtain samples from $p\left(\mathbf{x}_{k}, \mathbf{x}_{k-1} \mid \mathbf{z}_{0: k}\right)$ :

1. Make a joint draw for $\left\{\mathbf{x}_{k}, \mathbf{x}_{k-1}\right\}$ using a MetropolisHastings step ,

2. Update successively some elements in $\mathbf{x}_{k}$ by using a series of Metropolis-Hastings-within-Gibbs.

\section{Causality-Driven Agent Ranking}

The so-called probabilistic approach to causality, which has reached maturity over the past two decades (see for ex- 
ample Pearl [20], Geffner [9] and Shoam [22] for an extensive overview), establishes a convenient framework for reasoning and inference of causal relations in complex structural models.

Many notions in probabilistic causality rely extensively on structural models and in particular on causal Bayesian networks which are normally referred to as simply causal networks (CN's). A CN is a directed acyclic graph compatible with a probability distribution that admits a Markovian factorization and certain structural restrictions [20].

\section{Causal Hierarchies}

In this work the term causal hierarchies refers to ranking of agents with respect to their cumulative effect on the actions of the remaining constituents in the system. The word "causal" here reflects the fact that our measure of distinction embodies the intensity of the causal relations between the agent under inspection and its counterparts. Adopting the information-theoretic standpoint, in which the links of a CN are regarded as information channels [7], one could readily deduce that the total effect of an agent is directly related to the local information flow entailed by its corresponding in and out degrees. To be more precise, the total effect of an agent is computed by summing up the associated path coefficients (obtained by any standard Bayesian network learning approach) of either inward or outward links. This concept is further illustrated in Fig. 2.
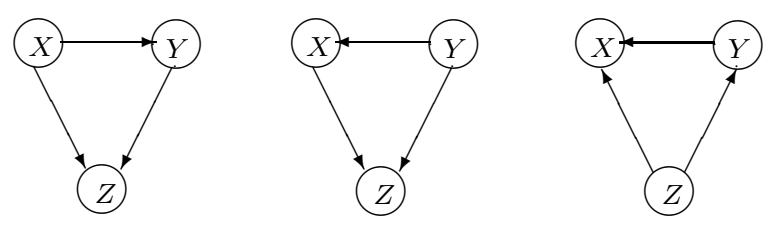

Figure 2. From left to right: depiction of the causal hierarchies (based on out degrees) $(X, Y, Z),(Y, X, Z)$, and $(Z, Y, X)$. The most influential agents in the causal diagrams from left to right are $X, Y$ and $Z$, respectively.

\section{Inferring Causal Hierarchies Via PCA}

To some extent, causal hierarchies can be inferred using the class of principal component analysis (PCA)-based methods. Probably the most promising one in the context of our problem is the multi-channel singular spectrum analysis (M-SSA), which is otherwise known as extended empirical orthogonal function (EEOF) analysis [11]. The novel approach we suggest has some relations with M-SSA. The relevant details, however, are beyond the scope of this work. A performance evaluation of both our method and M-SSA is provided in the numerical study part in the following sections.

\subsection{Structural Dynamic Modeling Approach}

Structural equation modeling is commonly used for representing the underlying links of a $\mathrm{CN}$ [20]. In our case, this formulation assumes a rather dynamic form (i.e., comprising of multiple time series of the agents' observed traits such as velocity and position)

$$
\mathbf{x}_{k}^{i}=\sum_{j \neq i} \sum_{m=1}^{p} \alpha^{j \rightarrow i}(m) \mathbf{x}_{k-m}^{j}+\epsilon_{k}^{i}, \quad i=1, . ., n
$$

where $\left\{\mathbf{x}_{k}^{i}\right\}_{k=0}^{\infty}$ and $\left\{\epsilon_{k}^{i}\right\}_{k=0}^{\infty}$ denote the $i$ th random process and a corresponding white noise driving sequence, respectively. The coefficients $\left\{\alpha^{j \rightarrow i}(m)\right\}_{m=1}^{p}$ quantify the causal influence of the $j$ th process on the $i$ th process. Notice that the Markovian model (5) has a finite-time horizon of the or$\operatorname{der} p$ (also referred to as the wake parameter). In the standard multivariate formulation, the coefficients $\alpha^{j \rightarrow i}(m)$ are square matrices of an appropriate dimension. For maintaining a reasonable level of coherency we assume that these coefficients are scalars irrespectively of the dimension of $\mathbf{x}_{\mathbf{k}}^{\mathbf{i}}$. Nevertheless, our arguments throughout this Section can be readily extended to the standard multivariate case.

The methodology underlying the so-called Granger causality [12] considers an F-test of the null hypothesis $\alpha^{j \rightarrow i}(m)=0, \quad m=1, \ldots, p$ for determining whether the $j$ th process G-causes the $i$ th process. The key idea here follows the simple intuitive wisdom that the more significant these coefficients are, the more likely they are to reflect a causal influence. In the framework of CNs the causal coefficients are related to the conditional dependencies within the probabilistic network, which in turn implies that their values can be learned based on the realizations of the time series $\left\{\mathbf{x}_{k}^{i}\right\}_{k=0}^{\infty}, \quad i=1, \ldots, n$. In what follows, we demonstrate how the knowledge of these coefficients allows us to infer the fundamental role of individual agents within the system. Before proceeding, however, we shall define the following key quantity.

Definition 1 (Causation Matrix) The causal influence of the process $\mathbf{x}^{j}$ on the process $\mathbf{x}^{i}$ can be quantified by

$$
A_{i j}=\sum_{m}\left[\alpha^{j \rightarrow i}(m)\right]^{2} \geq 0 .
$$

In the above definition, $A_{i j}$ denotes the coefficient relating the two processes $\mathbf{x}^{j}$ and $\mathbf{x}^{i}$ so as to suggest an overall matrix structure that would provide a comprehensive picture of the causal influences among the underlying processes. The matrix $A=\left[A_{i j}\right] \in \mathbb{R}^{n \times n}$, termed the causation matrix, essentially quantifies the intensity of all possible causal influences within the system (note that according to the definition of a $\mathrm{CN}$, the diagonal entries in $A$ vanish). It can be easily recognized that a single row in this matrix exclusively represents the causal interactions affecting each individual 
process. Similarly, a specific column in $A$ is comprised of the causal influences of a single corresponding process on the entire system. This premise motivates us to introduce the notion of total causal influence.

Definition 2 (Total Causal Influence Measure) The total causal influence (TCI) $T_{j}$ of the process $\mathbf{x}_{k}^{j}$ is obtained as the $l_{1}$-norm of the jth column in the causation matrix $A$, that is

$$
T_{j}=\sum_{i=1}^{n} A_{i j} .
$$

Having formulated the above concepts we are now ready to elucidate the primary contributions of this work, both of which rely on the TCI measure defined above.

\subsection{Dominance and Similarity}

A rather intuitive, but nonetheless striking, observation about the TCI is that it essentially reflects the dominance of each individual process in producing the underlying emergent behaviour. This allows us to decompose any complex act into its prominent behavioural building blocks (processes) using a hierarchical ordering of the form

$$
\text { Least dominant } T_{j_{1}} \leq T_{j_{2}} \leq \ldots \leq T_{j_{n}} \text { Most dominant }
$$

Equation (8) is given an interesting interpretation in the application part of this work, where the underlying processes $\left\{\mathbf{x}_{k}^{j}\right\}_{j=1}^{n}$ correspond to the motion of individual agents within a group. In the context of this example, the dominance of an agent is directly related to its leadership capabilities. By using the TCI measure it is therefore possible to distinguish between leaders and followers.

Another interesting implication of the TCI is exemplified in the following argument. Consider the two extreme processes in (8), one of which is the most dominant, $\mathbf{x}_{k}^{j_{n}}$, while the other is the least dominant, $\mathbf{x}_{k}^{j_{1}}$. Now, suppose we are given a new process $\mathbf{x}_{k}^{i}, \quad i \neq j_{1}, j_{n}$ and are asked to assess its dominance based exclusively on the two extremals, with respect to the entire system. Then, a common intuition would suggest to categorize $\mathbf{x}_{k}^{i}$ as a dominant process in the system whenever it resembles $\mathbf{x}_{k}^{j_{n}}$ more than $\mathbf{x}_{k}^{j_{1}}$ in the sense of $\left|T_{j_{n}}-T_{i}\right|<\left|T_{j_{1}}-T_{i}\right|$ and vice versa. This idea is summarized below.

Definition 3 (Causal Similarity) A process $\mathbf{x}_{k}^{j}$ is said to resemble $\mathbf{x}_{k}^{i}$ more than $\mathbf{x}_{k}^{l}$ if and only if $\left|T_{j}-T_{i}\right|<\mid T_{j}-$ $T_{l} \mid$.

In the context of the previously-mentioned example, we expect that dominant agents with high leadership capabilities would possess similar TCIs that would distinguish them from the remaining agents, the followers.

\subsection{Bayesian MCMC Estimation of $\alpha^{j \rightarrow i}$}

In typical applications the coefficients $\alpha^{j \rightarrow i}(m), m=$ $1, \ldots, p$ in (5) may be unknown. Providing that the realizations of the underlying processes are available it is fairly simple to estimate these coefficients by treating them as regressors. Such an approach by no means guarantees an adequate recovery of the underlying causal structure (see the discussion about the identifiability of path coefficients and a related assertion concerning non-parametric functional modeling in [20] pp. $156-157$, both have a clear connotation to the "fundamental problem of causal inference" [15]). Nevertheless, it provides a computationally efficient framework for making inference in systems with exceptionally large number of components. This premise is evident by noting from (5) that while fixing $i$ the coefficients $\alpha^{j \rightarrow i}(m), \quad \forall j \neq i, m=1, \ldots, p$ are statistically independent of $\alpha^{j \rightarrow l}(m), \quad \forall l \neq i$.

In a Bayesian framework we confine the latent causal structure by imposing a prior on the coefficients $\alpha^{j \rightarrow i}(m)$. Let $p_{\alpha}^{i}$ and $p_{\alpha}^{j \rightarrow i}$ be the priors of $\left\{\alpha^{j \rightarrow i}(m), \forall j \neq i\right\}$, and $\alpha^{j \rightarrow i}(m)$, respectively. Let also $p_{\epsilon}^{i}$ be some prescribed (not necessarily Gaussian) probability density of the white noise in (5). Then,

$$
\begin{aligned}
& p\left(\left\{\alpha^{j \rightarrow i}(m), \forall j \neq i\right\} \mid \mathbf{x}_{0: k}^{1: n}\right) \propto \\
& p_{\alpha}^{i} \prod_{t=p}^{k} p\left(\mathbf{x}_{t}^{i} \mid\left\{\alpha^{j \rightarrow i}(m), \mathbf{x}_{t-p: t-1}^{j}, \forall j \neq i\right\}\right) \\
= & p_{\alpha}^{i} \prod_{t=p}^{k} p_{\epsilon}^{i}\left(\mathbf{x}_{t}^{i}-\sum_{j \neq i} \sum_{m=1}^{p} \alpha^{j \rightarrow i}(m) \mathbf{x}_{t-m}^{j}\right), \quad i=1, \ldots, n
\end{aligned}
$$

where $\mathbf{x}_{0: k}^{1: n}=\left\{\mathbf{x}_{0}^{1}, \ldots, \mathbf{x}_{0}^{n}, \ldots, \mathbf{x}_{k}^{1}, \ldots, \mathbf{x}_{k}^{n}\right\}$, and $\mathbf{x}_{t-p: t-1}^{j}=\left\{\mathbf{x}_{t-p}^{j}, \ldots, \mathbf{x}_{t-1}^{j}\right\}$. A viable estimation scheme for $\alpha^{j \rightarrow i}(m)$ which works well in most generalized settings is a Metropolis-within-Gibbs sampler that operates either sequentially or concurrently on the conditionals

$$
\begin{aligned}
p\left(\alpha^{j \rightarrow i}(m) \mid \mathbf{x}_{0: k}^{1: n},\left\{\alpha^{l \rightarrow i}, \forall l \neq j, i\right\}\right) \propto \\
p_{\alpha}^{j \rightarrow i} \prod_{t=p}^{k} p\left(\mathbf{x}_{t}^{i} \mid\left\{\alpha^{l \rightarrow i}(m), \mathbf{x}_{t-p: t-1}^{l}, \forall l \neq i\right\}\right)
\end{aligned}
$$

The obtained estimates at time $k$ are then taken as the average of the converged chain (i.e., subsequent to the end of some prescribed burn-in period).

\subsection{Causal Reasoning in Cluttered Environments}

In many practical applications the constituent underlying traits, which are represented here by the processes $\left\{\mathbf{x}_{k}^{j}\right\}_{j=1}^{n}$, may not be perfectly known (in the context of our work these could be the object position and velocity, $\boldsymbol{\mu}_{k}^{j}, \dot{\boldsymbol{\mu}}_{k}^{j}$ ). 
Hence instead of the actual traits one would be forced to use approximations that might not be consistent estimates of the original quantities (e.g., $\hat{\boldsymbol{\mu}}_{k}^{j}, \hat{\dot{\boldsymbol{\mu}}}_{k}^{j}$ ). As a consequence, the previously suggested structure might cease being an adequate representation of the latent causal mechanism. A plausible approach for alleviating this problem is to introduce a compensated causal structure that takes into account the exogenous disturbances induced by the possibly inconsistent estimates. Such a model can be readily formulated as a modified version of (5), that is

$$
\hat{\boldsymbol{\mu}}_{k}^{i}=\sum_{j \neq i} \sum_{m=1}^{p} \alpha^{j \rightarrow i}(m) \hat{\boldsymbol{\mu}}_{k-m}^{j}+\boldsymbol{\epsilon}_{k}^{i}+\boldsymbol{\zeta}_{k}^{i}, \quad i=1, . ., n
$$

where the additional factor $\boldsymbol{\zeta}_{k}^{i}$ denotes an exogenous bias. Hence, one can use (11) to predict the effects of interventions in $\boldsymbol{\zeta}_{k}^{i}$ directly from passive observations (which are taken as an output of a tracking algorithm, e.g., $\hat{\boldsymbol{\mu}}_{k}^{j}$ or $\hat{\dot{\boldsymbol{\mu}}}_{k}^{j}$ ) without adjusting for confounding factors. See [20] (p. 166) for further elaborations on the subject.

\section{Illustrative Examples}

We demonstrate the performance of our suggested reasoning methodology and some of the previously mentioned concepts using both synthetic and realistic examples. All the scenarios considered here involve a group of dynamic agents, some of which are leaders that behave independently of all others. The leaders themselves may exhibit a highly nonlinear and non-predictive motion pattern which in turn affects the group's emergent behaviour. We use a standard CN (5) with a predetermined time horizon $p$ for disambiguating leaders from followers based exclusively on their instantaneous TCIs. In all cases the processes $\mathbf{x}_{k}^{i}$, $i=1, \ldots, n$ are taken as either the increment $\dot{\boldsymbol{\mu}}_{k}^{i}$ or position $\boldsymbol{\mu}_{k}^{i}$ of each individual agent in the group. In addition, the unified tracking and reasoning paradigm is demonstrated by replacing the actual position and increment with the corresponding outputs of the MCMC cluster tracking algorithm, $\hat{\dot{\mu}}_{k}^{i}$ and $\hat{\boldsymbol{\mu}}_{k}^{i}$.

The performance of the causality inference scheme is directly related to its ability to classify leaders based on their TCI values. As leaders are, by definition, more dominant than followers in some measure space, essentially shaping the overall group behaviour, we expect that their TCI values would reflect this fact. Furthermore, the hierarchy (8) should allow us to disambiguate them from the remaining agents according to the notion of causal similarity which was introduced in Section 3.2. Following this argument we define a rather distinctive performance measure which allows us to assess the aforementioned qualities.

Let $G$ be a set containing the leaders indices, i.e., $G=\left\{j \mid x_{k}^{j}\right.$ is a leader's instantaneous position or velocity $\}$.
Let also $\boldsymbol{v}$ be a vector containing the agents' ordered indices according to the instantaneous hierarchy at time $k$

$$
T_{j_{1}} \leq \cdots \leq T_{j_{n}}
$$

i.e., $\boldsymbol{v}=\left[j_{n}, \ldots, j_{1}\right]^{T}$. Having stated this we can now define the following performance index

$$
e=\arg \max _{i \in[1, n]}\left(\boldsymbol{v}_{i} \in G\right) .
$$

The above quantity indicates the worst TCI ranking of a leader. As an example, consider a case with, say, 5 leaders. Then the best performance index we could expect would be 5 , implying that all leaders have been identified and were properly ranked according to their TCIs. If the performance index yields a value greater than 5, say 10, it implies that all leaders are ranked among the top 10 agents according to their TCIs. The performance index cannot go below the total number of leaders and cannot exceed the total number of agents.

\section{Swarming of Multiple Interacting Agents (Boids)}

Our first example pertains to identification of leaders and followers in a dynamical system of multiple interacting agents, collectively performing in a manner usually referred to as swarming or flocking.

In the current example, Reynolds-inspired flocking [21] is used to create a complex motion pattern of multiple agents. Among these agents, there are leaders, who independently determine their own position and velocity, and followers, who interact among themselves and follow the leader agents.

The inference scheme performance over 100 Monte Carlo runs, in which the agents initial state and velocity were randomly picked, is provided in Fig. 3. The synthetic scenario considered consists of 30 agents, 4 of which are actual leaders. The performance index cumulative distribution function $(\mathrm{CDF})$ for this scenario, which is illustrated via the 50,70 and 90 percentile lines, is shown over the entire time interval in the left panel in this figure. The percentiles indicate how many runs out of 100 yielded a performance index below a certain value. Thus, 50 percent of the runs yielded a performance index below the 50 percentile, 70 percent of the runs attained values below the 70 percentile, and so on. Following this, it can be readily recognized that from around $k=150$ the inference scheme is able to accurately identify the actual leaders in 50 percent of the runs. A further examination of this figure reveals that the 4 actual leaders are ranked among the top 6 from around $k=180$ in 90 percent of the runs.

A comparison of leaders ranking capabilities of the proposed approach with that of the M-SSA method is provided in the right panel in Fig. 3. The instantaneous CDFs of both 
techniques are shown when using either position or velocity time series data. This figure clearly demonstrates the superiority of the proposed approach with respect to the M-SSA.

\section{Identifying Extended Leaders in Clutter}

In the following example the actual agent tracks are replaced by the output of an MCMC-based tracking approach that was initially derived in $[6,19]$ and is briefly described in Section 2. The scenario consists of 4 agents out of which 2 are leaders. As before we use the Boids system for simulating the entire system. This time, however, the produced trajectories are contaminated with clutter and additional points representing multiple emissions from possibly the same agent (i.e., agents are assumed to be extended objects). These observations are then used by the MCMC tracking algorithm of which the output is fed to the causality detection scheme, in a fashion similar to the one described in Section 3.4.

The tracking performance of the MCMC algorithm is demonstrated both in Fig. 4 and in the left panel in Fig. 5. In Fig. 4, the estimated tracks and the cluttered observations are shown for a typical run. The averaged tracking performance of the MCMC approach is further illustrated based on 20 Monte Carlo runs using the Hausdorff distance [6] in Fig. 5. From this Figure it can be seen that the mean tracking errors become smaller than 1 after approximately 50 time steps in either cases of cluttered and non-cluttered observations.

The averaged leaders ranking performance in this example is illustrated for 3 different scenarios in the right panel in Fig. 5. Hence, it can be readily recognized that the two leaders are accurately identified after approximately 10 time steps when the agent positions are perfectly known. As expected, this performance is deteriorated in the presence of clutter and multiple emissions, essentially attaining an averaged ranking metric of nearly 2.5 after 60 time steps.

\section{Identifying Group Leaders from Video Data}

Our third, more practical example, deals with the following application. Consider a group of people, among which there are subgroups of leaders and followers. The followers coordinate their paths and motion with the leader. Using video observations only of the group, determine who the group leaders are. To that end, one must first develop a procedure for estimating the trajectories of $n$ people from a given video sequence. The input to the described procedure is a movie with $n$ moving people, where $n$ is known. The objective is to track each person along the frame sequence, and then feed this information into the $\mathrm{CN}$ mechanism for inferring the leaders and followers.

As we are dealing with a rather noiseless and noncluttered scenario, a simple k-means clustering was used to recover individual person tracks from SIFT (scale-invariant feature transform) features. This approach was applied to two different video sequences in which there were 5 followers and 1 leader. Snapshots are shown in the upper panel in Fig. 6. In these videos, the actual leader (designated by a red shirt) performs a random trajectory, and the followers loosely follow its motion pattern. The clustering procedure described above is used to estimate the trajectories of the objects (the trajectories were filtered using a simple moving-average procedure to reduce the amount of noise contributed by the k-means clustering method). These trajectories were fed into the causality inference scheme.

The results of this procedure are shown in the bottom panel in Fig. 6, which depicts the causality performance index for two values of the finite-time horizon (wake parameter), $p$. It is clearly seen that from a certain time point the algorithm identifies the actual leader in both videos irrespective to the value of $p$.

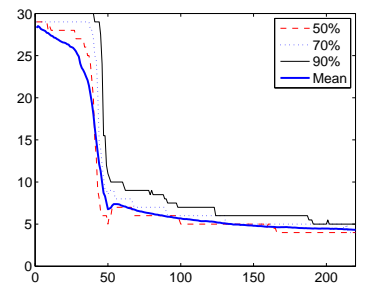

(a) Causal Ranking

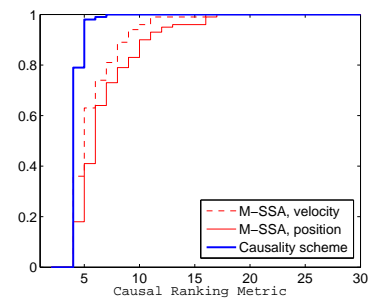

(b) Causal Ranking CDF
Figure 3. Identification performance over time (abscissa) of the causality scheme (left) and the ranking CDF at time $t=220$ (right) of both the causality scheme and the M-SSA method (using either velocity or position data) based on 100 Monte Carlo runs.

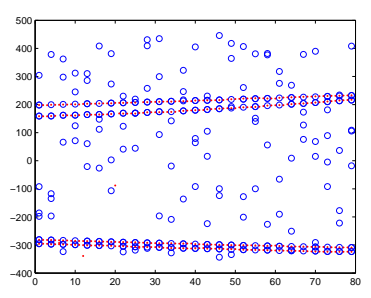

(a) $\mathrm{X}$

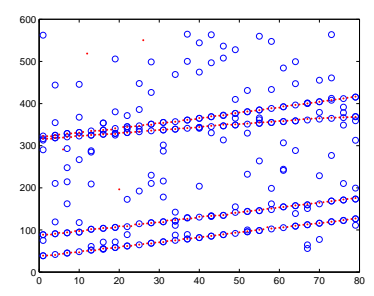

(b) $\mathbf{Y}$
Figure 4. Point observations and estimated tracks over time (abscissa).

\section{Concluding Remarks}

A novel causal reasoning framework has been proposed for ranking agents with respect to their contribution in shaping the collective behaviour of the system. The proposed scheme copes with clutter and multiple emissions from extended agents by employing a Markov chain Monte Carlo 


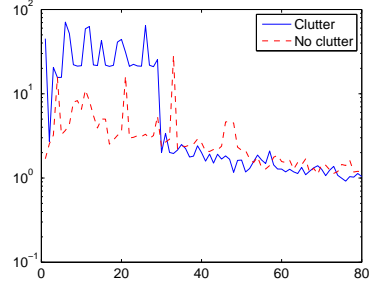

(a) Hausdorff Distance

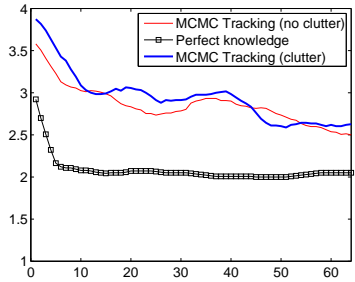

(b) Causality Ranking
Figure 5. Tracking performance and causality ranking over time (abscissa) averaged over 20 Monte Carlo runs.

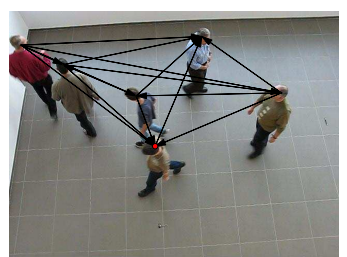

(a) Video 1

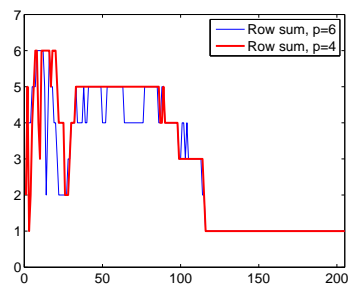

(c) Video 1

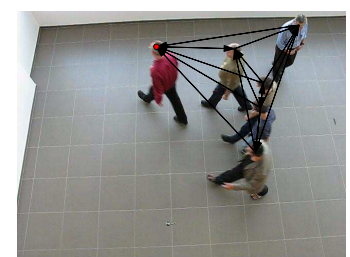

(b) Video 2

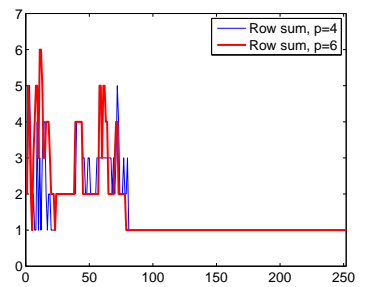

(d) Video 2
Figure 6. Reconstructed instantaneous causal diagrams shown with the corresponding video frames (upper panel), and causality ranking performance over time (lower panel).

group tracking method. This approach has been successfully applied for identifying leaders in groups in both synthetic and realistic scenarios.

\section{References}

[1] R. Albert and A.-L. Barabsi. Statistical mechanics of complex networks. Reviews of Modern Physics, 74(1):47-97, 2002. 2

[2] S. Ali and M. Shah. Floor fields for tracking in high density crowd scenes. In Computer Vision ECCV 2008, volume 5303 of Lecture Notes in Computer Science, pages 114. Springer Berlin / Heidelberg, 2008. 2

[3] D. Angelova and L. Mihaylova. Extended object tracking using Monte Carlo methods. IEEE Transactions on Signal Processing, 56(2):825-832, 2008. 2

[4] M. Arulampalam, S. Maskell, N. Gordon, and T. Clapp. A tutorial on particle filters for online nonlinear/non-Gaussian
Bayesian tracking. IEEE Trans. on Signal Proc., 50(2):174188, 2002. 2

[5] C. Berzuini, G. Nicola, W. R. Gilks, and C. Larizza. Dynamic Conditional Independence Models and Markov Chain Monte Carlo Methods. Journal of the American Statistical Association, 92(440):1403-1412, 1997. 2

[6] A. Carmi, F. Septier, and S. J. Godsill. The Gaussian mixture MCMC particle algorithm for dynamic cluster tracking. pages 1179-1186. 12th International Conference on Information Fusion, July 2009. 2, 3, 7

[7] J. Cheng, R. Greiner, J. Kelly, D. Bell, and W. Liu. Learning Bayesian network from data: An information-theory based approach. Artificial Intelligence, 1-2:43-90, 2002. 4

[8] S. N. Dorogovtsev and J. F. F. Mendes. Evolution of networks. Advances in Physics, 51:1079-1187, 2002. 2

[9] H. Geffner. Default Reasoning: Causal and Conditional Theories. MIT Press, 1992. 4

[10] A. Gning, L. Mihaylova, S. Maskell, S. K. Pang, and S. Godsill. Group object structure and state estimation with evolving networks and Monte Carlo methods. IEEE Transactions on Signal Processing, 12(2):523-536, 2011. 2

[11] N. Golyandina, V. Nekrutkin, and A. Zhigljavsky, editors. Analysis of Time Series Structure: SSA and related techniques. Chapman and Hall, 2001. 4

[12] C. W. J. Granger. Investigating causal relations by econometric models and cross-spectral methods. Econometrica, 37:424-438, 1969. 1, 4

[13] P. J. Green. Reversible Jump Markov Chain Monte Carlo Computation and Bayesian Model Determination. Biometrika, 82(4):711-732, December 1995. 2

[14] D. Helbing. Traffic and related self-driven many-particle systems. Review of Modern Physics, 73:1067-1141, 2002. 2

[15] P. W. Holland. Statistics and causal inference. Journal of the American Statistical Association, 81:945-960, 1986. 5

[16] Z. Khan, T. Balch, and F. Dellaert. MCMC-based particle filtering for tracking a variable number of interacting targets. IEEE Transactions on Pattern Analysis and Machine Intelligence, 27(11):1805-1819, Nov. 2005. 2

[17] R. Mahler. Statistical Multisource-multitarget Information Fusion. Artech House, Boston, 2007. 2, 3

[18] L. Mihaylova, R. Boel, and A. Hegyi. Freeway traffic estimation within recursive Bayesian framework. Automatica, 43(2):290-300, February 2007. 2

[19] S. K. Pang, J. Li, and S. J. Godsill. Detection and tracking of coordinated groups. IEEE Transactions on Aerospace and Electronic Systems, 47(1):472 - 502, 2011. 2, 3, 7

[20] J. Pearl. Causality: Models, Reasoning, and Inference. Cambridge University Press, 2000. 1, 4, 5, 6

[21] C. W. Reynolds. Flocks, herds, and schools: A distributed behavioral model. Computer Graphics, 21:25-34, 1987. 1, 6

[22] Y. Shoam. Reasoning About Change: Time and Causation from the Standpoint of Artificial Intelligence. MIT Press, 1988. 4

[23] B. Vo, S. Singh, and A. Doucet. Sequential Monte Carlo Methods for Multi-Target Filtering with Random Finite Sets. IEEE Transactions on Aerospace and Electronic Systems, 41(4):1224-1245, October 2005. 2 